\title{
Individual vocal signatures show reduced complexity following invasion
}

Grace Smith-Vidaurre ${ }^{1}$, Valeria Perez ${ }^{1}$, and Timothy F. Wright ${ }^{1}$

${ }^{1}$ Department of Biology, New Mexico State University, Las Cruces, NM, USA

\section{Corresponding author:}

Grace Smith-Vidaurre

Department of Biology

1200 S. Horseshoe Dr.

New Mexico State University

Las Cruces, NM, USA 88003

gsvidaurre@gmail.com

575-646-4863

ORCID: 0000-0002-0155-8159

Abstract: The manner in which vocal learning is used for social recognition may be sensitive to the social environment. Biological invaders capable of vocal learning are useful for testing this possibility, as invasion alters population size. If vocal learning is used for individual recognition, then individual identity should be encoded in frequency modulation patterns of acoustic signals. Furthermore, frequency modulation patterns should be more complex in larger social groups, reflecting greater selection for individual distinctiveness. We compared social group sizes and used supervised machine learning and frequency contours to compare contact call structure between native range monk parakeets (Myiopsitta monachus) in Uruguay and invasive range populations in the U.S. Invasive range sites exhibited fewer nests and simpler frequency modulation patterns. Beecher's statistic revealed reduced individual identity content and fewer possible unique individual signatures in invasive range calls. Lower estimated social densities and simpler individual signatures are consistent with relaxed selection on the complexity of calls learned for individual recognition in smaller social groups. These findings run counter to the traditional view that vocal learning is used for imitation, and suggest that vocal learning can be employed to produce individual vocal signatures in a manner sensitive to local population size. 
35 Introduction: One way in which vocal learning can be used is to signal group identity for

36 social recognition [1-3]. Patterns of acoustic convergence within social groups, consistent with vocal learning being employed for group recognition, have been identified in cetaceans, bats, songbirds, and parrots [3]. Vocal learning may also be used to create individually distinctive acoustic signals, often termed "individual signatures", as found in many of the same taxonomic groups [1,4-6]. The manner in which different taxa use vocal learning to recognize group members could be sensitive to population size and social dynamics. In larger populations, greater social density results in more individuals for potential

43 receivers to discriminate, leading to increased uncertainty about signalers' identities, and

44 increased selection on signalers to produce distinctive individual signatures [7]. In species

45 that employ vocal learning for individual vocal recognition, such selection to produce

46 individually distinctive signals should manifest in the acoustic structure of learned calls. For

47 instance, learning can be employed to produce individually distinctive frequency modulation

48 patterns in acoustic signals [4-6,8]. In such systems, frequency modulation patterns in

49 learned signals should be more complex in larger social groups, and simpler in smaller social

50 groups, leading to fewer potential unique individual signatures [9]. Biological invaders offer

51 useful models for addressing these ideas, as invasive populations often exhibit reduced

52 population sizes compared to the native range [10].

We asked whether the way in which vocal learning is employed for social recognition is

54 resilient or sensitive to changes in population size by evaluating contact calls of an invasive

55 parrot. Monk parakeets (Myiopsitta monachus) are native to South America, may use vocal

56 learning for individual recognition [11], and have established invasive populations across the 57 world through the pet trade [12]. We predicted that estimated social densities would be lower 
58 following invasion, and frequency modulation patterns in invasive range contact calls would

59 be simpler compared to the native range.

60

\section{Methods}

62 Contact call recording

63 Native range contact calls were recorded in 2017 at nest sites in Uruguay, as previously

64 described [11]. Invasive range contact calls were recorded at nest sites across five states in

65 the U.S. over different years. When possible, we estimated the numbers of nests visible at

66 recording sites (Supplementary Table 1). We obtained previously published calls recorded in

672004 in Connecticut, Florida, Louisiana, and Texas [13]. Calls were also recorded in Texas

68 and New Orleans in 2011, Arizona in 2018, and Texas in 2019. We repeatedly sampled

69 contact calls from 8 native and 9 invasive birds, otherwise, a single contact call was obtained

70 per unmarked bird (Supplementary Tables 2 - 4). Most recording sessions were performed

71 with Marantz PMD661 MKII and PMD660 solid state recorders, Sennheiser ME67 long

72 shotgun microphones, and digitized at $44100 \mathrm{~Hz}$ sampling rate and 16 bit depth. Invasive

73 range 2004 sessions employed Marantz PMD670 or PMD690 recorders with Sennheiser

74 ME67/K6 shotgun microphones, digitized at $48000 \mathrm{~Hz}$ and 16 bits [13]. Invasive range calls

75 were selected using Raven version 1.4 [14], and pre-processing was performed with the

76 warbleR package in $\mathrm{R}$ to retain high quality calls $[15,16]$.

78 Acoustic structure analyses

79 Differences in call structure between ranges were evaluated with supervised machine

80 learning models that classified calls back to each range. Models were built with 203

81 predictors, including 15 standard acoustic measurements and 188 features (Supplementary

82 Methods 2.1.1). Spectrum-based measurements were obtained using a Hanning window, 
83 window length of 398, window overlap of 90 for Fourier transformations, and a bandpass filter

84 of 0.5 to $9 \mathrm{kHz}$ [11]. 1561 calls were split into training, validation, and prediction datasets. 548

85 calls used for prediction (230 native, 318 invasive) were visualized in two-dimensional

86 acoustic space by applying multidimensional scaling (MDS) to the proximity matrix of the final

87 random forests model. A Gaussian kernel density estimator was applied to MDS coordinates

88 to yield density in random forests acoustic space.

80 calls were subsampled to evaluate frequency modulation patterns between ranges.

9010 sites were randomly selected per range, and 4 calls randomly chosen per site.

91 Fundamental frequencies were estimated at 100 timepoints per call, and used to manually

92 trace the second harmonic with warbleR [15]. 5 points were dropped from the start and end of

93 each frequency contour, and spline interpolation performed across the remaining 90 points

94 with smoothing. Smoothed contours were used to estimate frequency peaks and troughs per

95 call. We obtained means and standard error of the number of peaks per call, modulation rate

96 (number of peaks divided by the duration of call), and the maximum slope of frequency

97 modulation per call (largest negative slope between peaks and neighboring troughs). The

98 effect size of range was calculated as Cohen's $d$ on the 3 frequency modulation

99 measurements, as well as 15 standard acoustic measurements. Acoustic measurements with

100 the largest effects of range were also compared among invasive calls sampled over time, to

101 assess whether the patterns identified held over 15 years of sampling in the invasive range.

102 Invasive range populations that grew over time could experience greater selection for more

103 distinctive individual signatures, confounding direct comparisons between ranges.

104

105 Individual identity content

106 Beecher's statistic (HS) was employed to quantify the amount of individual identity content in

107 calls of repeatedly sampled individuals per range [17]. We used 5 individuals per range, 
108 recorded at a single site-year in the native range (site 1145 in 2017), or a single city-year

109 (Austin, TX in 2019) in the invasive range. These individuals showed similar patterns of

110 dispersion in acoustic space (Supplementary Figure 2). HS was calculated by principal

111 components analysis on Mel-frequency cepstral coefficients (MFCC) of all calls per individual,

112 or the second harmonic frequency contours for 5 randomly sampled calls per bird (or all calls

113 if 5 or less were recorded), with 5 points dropped on either end, and without spline

114 interpolation. The number of possible unique individual signatures given the amount of

115 individual identity content per range was estimated as $2^{\mathrm{HS}}[9,17]$.

\section{Results}

\section{Lower nest density in invasive range}

119 We observed (mean \pm standard error) $36.35 \pm 12.24$ nests per site for the native range, and

$1205.94 \pm 1.23$ for the invasive range. The maximum number of nests observed at a given site

121 was an order of magnitude greater in the native range (Supplementary Table 1), and nest

122 estimates were significantly different between ranges (Mann-Whitney difference in location

123 with 95\% Cl: $14(7,26), Z=4.21, p<0.0001)$.

125 Simpler frequency modulation patterns in invasive range calls

126 Native and invasive range calls exhibited structural differences. Frequency modulation

127 patterns were visibly different between ranges (Figure 1a), consistent with high classification

128 accuracy by supervised random forests (Supplementary Table 5), and differentiation between

129 ranges in random forests acoustic space (Figure 1b). Frequency modulation patterns

130 contributed significantly to structural differences between ranges. Invasive range calls

131 exhibited fewer frequency modulation peaks, lower modulation rates, and shallower maximum

132 peak - trough slopes (Figure 2b). The effects of range on frequency modulation 
bioRxiv preprint doi: https://doi.org/10.1101/2020.09.02.280297; this version posted September 3, 2020. The copyright holder for this preprint (which was not certified by peer review) is the author/funder, who has granted bioRxiv a license to display the preprint in perpetuity. It is made available under aCC-BY-NC-ND 4.0 International license.

133 measurements were large and significant (Supplementary Table 6), and these trends were

134 consistent over the 15 year sampling period in the invasive range (Supplementary Figure 1).

135

136 Figure 1:
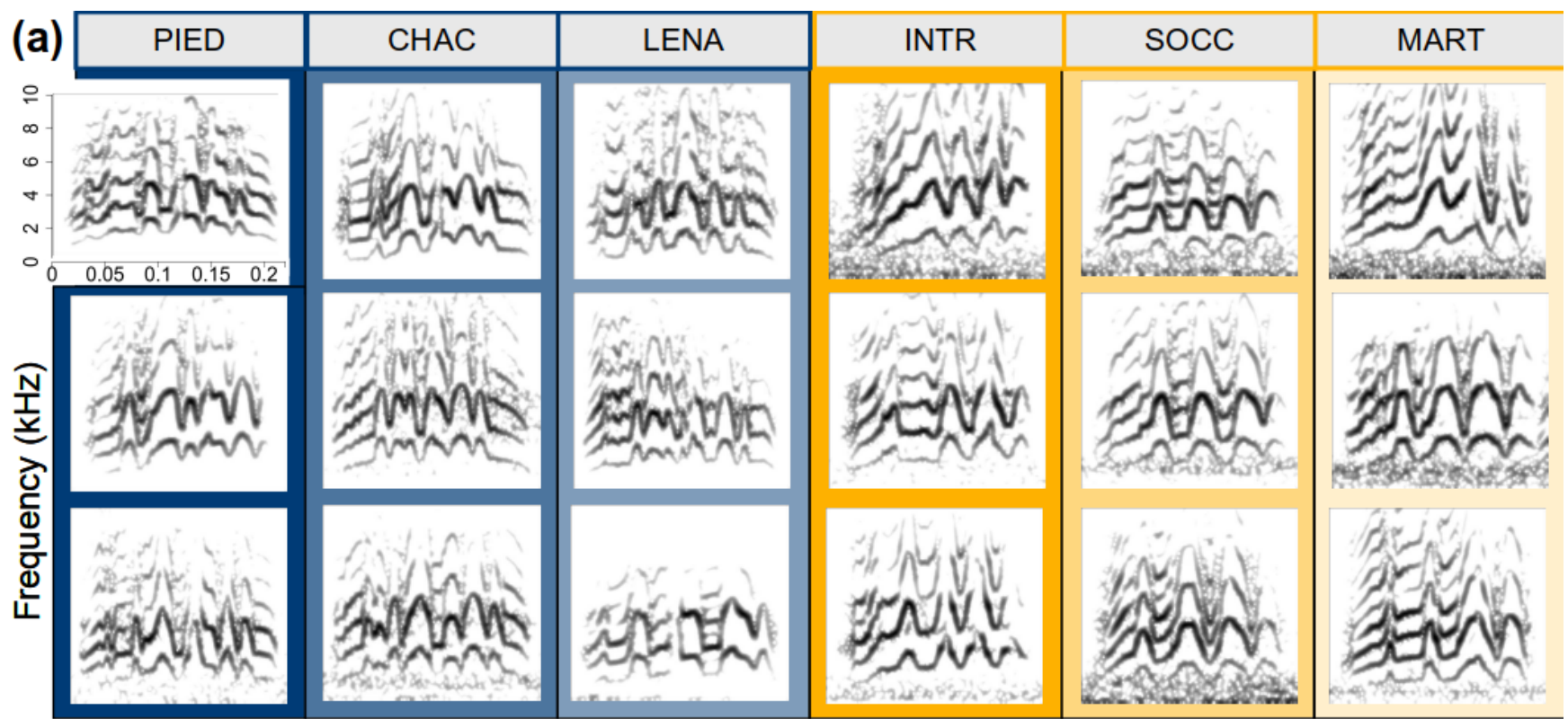

Time (s)
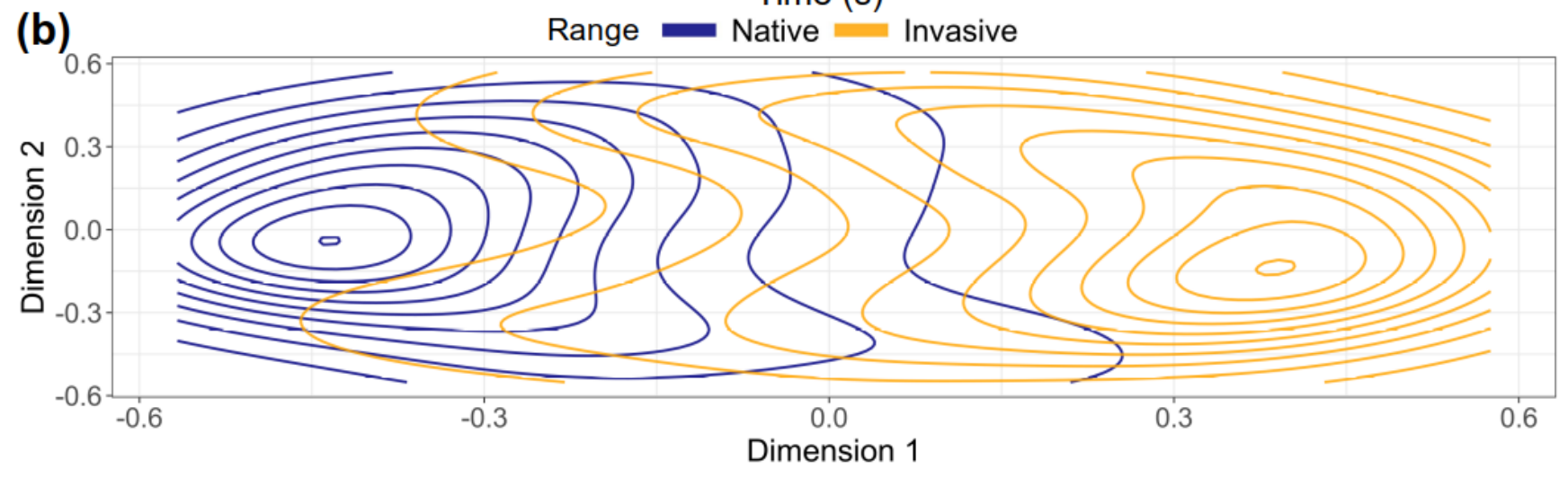

137 Figure 1 Legend: Differentiation in contact call structure between ranges. (a) Example lexicon

138 with spectrograms of 3 randomly selected calls from 3 sites per range, each call represents a 139 different unmarked individual. Calls were sampled over similar areas per range. Native and

140 invasive range calls shown were recorded in 2017 and 2019, respectively. (b) Estimated 141 kernel density contours in random forests acoustic space for the prediction dataset. Contours 142 delineate bins of density values, with each bin representing 1/10th of the density per range.

143 Dimension 1 coordinates were flipped to place native range contours on the left-hand side. 
144 Figure 2:
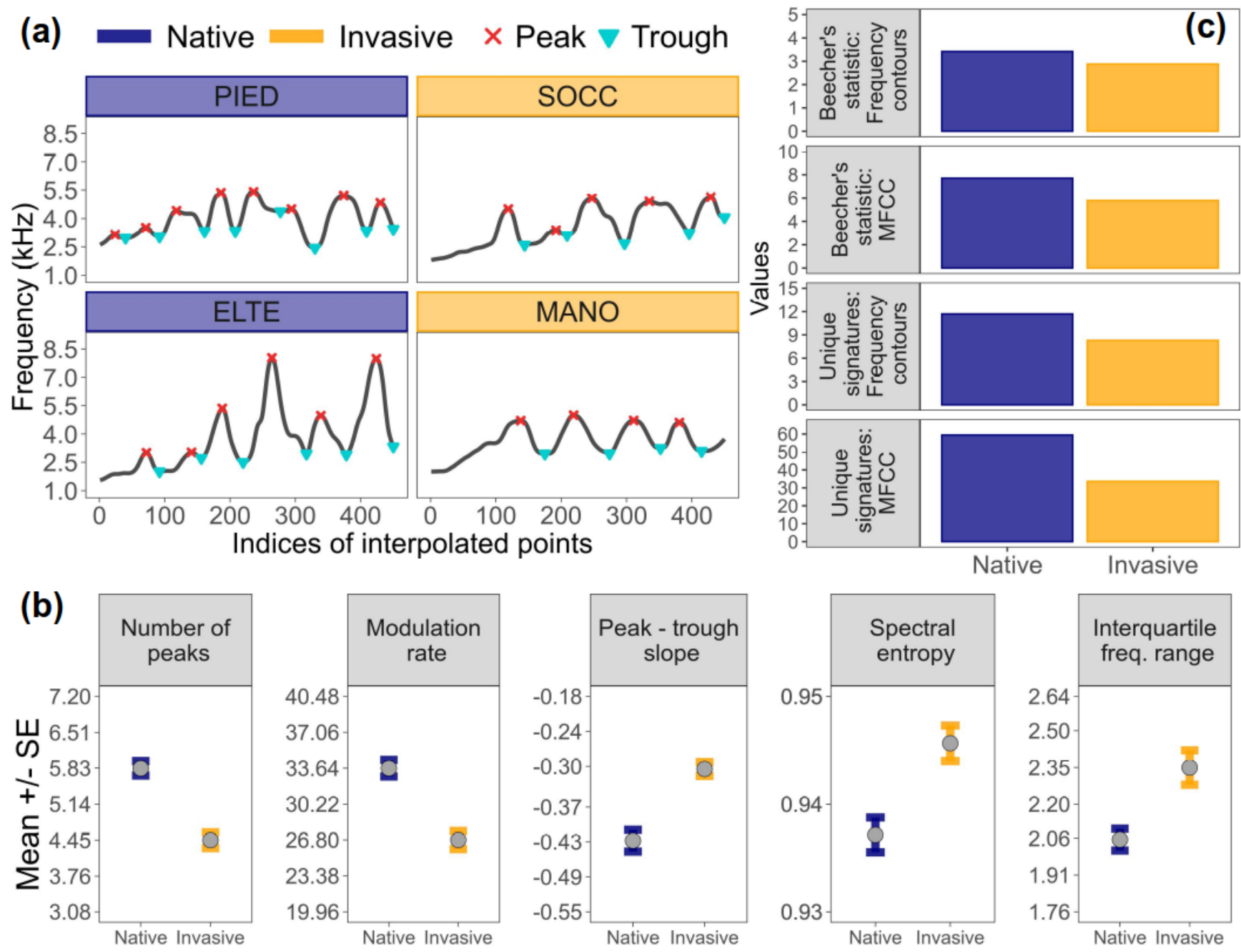

146 Figure 2 Legend: Simpler frequency modulation patterns in the invasive range. (a) Smoothed second harmonic frequency contours, marked with estimated peaks and troughs. (b) Mean and standard error of acoustic measurements with the largest effects of range, in decreasing order of absolute effect size magnitude (left to right). (c) Beecher's statistic and the number of possible unique individual signatures, calculated with frequency contours as well as Mel-

151 frequency cepstral coefficients. 
152

153 Beecher's statistic was lower for invasive range calls. This reduced individual identity content yielded fewer distinctive individual signatures compared to native range calls, and trends were similar between MFCC and frequency contours (Figure 2c, Supplementary Table 7). MFCC

156 includes frequency modulation patterns as well as other aspects of acoustic structure, 157 including timbre and absolute frequency, that may arise from individual differences in vocal 158 morphology.

160 Discussion: Social group sizes and contact calls of native and invasive range monk 161 parakeets were compared to ask whether the use of vocal learning for individual recognition could be sensitive to changes in the social environment following invasion. We found smaller social groups at invasive range sites, and frequency modulation patterns, which can be altered by learning and used for individual vocal recognition [4-6,8], were significantly simpler and contained less individual identity content in invasive range calls. Our results suggest that monk parakeets use vocal learning for individual recognition, and that this use of vocal learning for social recognition is sensitive to social changes associated with invasion. social densities and hence relaxed selection for individual vocal distinctiveness. Smaller

170 invasive population sizes are a well-documented outcome of invasion [10]. Indeed, we

171 observed fewer nests at invasive range sites, indicative of reduced local social densities

172 compared to the native range. We do not know whether social dynamics are also altered

173 following invasion, but this seems plausible given reduced population sizes as well as

174 increased population isolation compared to the population contiguity observed in the native 175 range (Smith-Vidaurre, pers. obs.). 
Alternatively, the structural changes we identified could be due to a withdrawal of

177 learning founder effect [18-20], if invasive populations were established by juvenile and/or

178 captive birds that lacked adult tutors and thus developed atypical calls. We consider this

179 alternative less likely, because changes in acoustic structure were concentrated on aspects of

180 frequency modulation rather than distributed across all acoustic measurements. Furthermore,

181 these changes were seen across multiple, presumably independent, invasions in 5 different

182 states. Another possibility is that structural change could be due to genetic bottlenecks,

183 another common outcome of biological invasions [21,22]. If structural variation in contact calls

184 had a strong genetic component, acoustic variation should have decreased in concert with the

185 reduced neutral genetic variation previously reported in the U.S. [23], yielding high overlap

186 among individuals in acoustic space over short geographic distances. Instead, we identified

187 similarly high levels of acoustic variation among individuals in the invasive range as

188 previously found in the native range, indicating that although invasive range calls contain less

189 individual identity information, individuals in both ranges are using learning to diverge in

190 acoustic space (Smith-Vidaurre et al., unpublished data).

Reduced individual identity content in invasive range calls is consistent with relaxed

192 selection for individual recognition in smaller populations. Beecher's statistic (HS) calculated

193 with Mel-frequency cepstral coefficients (MFCC) estimated 59 unique individual signatures for

194 the native range versus 33 for the invasive range, while HS from frequency contours

195 estimated 11 versus 8 unique signatures. Future work should manipulate social group size as

196 well as social dynamics to ask whether vocal learning facilitates altering individual signatures

197 to match changes in social group complexity over short timescales. Monk parakeets exhibit

198 high fission-fusion fluidity [24], but how social dynamics influence individual recognition

199 remains an open question. 
201 developmental constraints. In larger social groups, receivers incur the cognitive costs of

202 discriminating among more individuals while simultaneously processing more complex

203 individual signatures against a noisier background. Signalers should also experience costs of

204 learning to encode more distinctive individual signatures through fine-scale structural

205 variation. Monk parakeets both produce and recognize contact calls, therefore all individuals

206 should experience costs of both receiver perception and vocal production [3]. As the

207 perception and production of complex individual signatures impose greater cognitive burdens,

208 simpler individual signatures should be present in smaller groups in which accurate

209 recognition is possible without such complexity. Simpler or less informative signaler traits

210 should also be favored when the costs of errors in individual recognition are lower [25]. Both

211 factors could be working in the smaller populations found in the invasive range to yield

212 simpler signatures. Finally, simpler individual signatures may reflect developmental

213 constraints in receiver perception. Although parrots are considered open-ended vocal learners

214 [26], we do not know whether auditory perception remains sensitive throughout adulthood

215 [27]. In monk parakeets, perception of individual signatures may be constrained by local

216 social densities experienced during sensitive developmental periods. The findings we present

217 here on complexity of individual signatures produced by an invasive parrot add to a

218 foundation for future work on how vocal learning is employed for individual recognition in

219 dynamic social groups. 
220 Funding: This research was supported by a Fulbright Study/Research grant to G.S.V., a New

221 Mexico State University Honors College scholarship to Clara Hansen, an American

222 Ornithologists' Union Carnes Award to G.S.V, Experiment.com crowdfunding led by G.S.V and

223 Dr. Kevin Burgio, a donation to G.S.V from Drs. Michael and Susan Achey, a NMSU Whaley

224 Field Award to G.S.V, and MARC funding to V.P. (Biomedical Research Training for Honor

225 Undergraduates supported by NIH/NIGMS 5T34GM007667).

226

227 Acknowledgments: We thank Clara Hansen for help trapping, marking and recording

228 parakeets in Uruguay as well as Tania Molina for help with Uruguay fieldwork. We thank

229 numerous additional people for their support throughout native range fieldwork, as

230 acknowledged in Smith-Vidaurre et al. (2020). We are grateful to Zoë Amerigian and

231 Alexandra Bicki for their help during 2019 fieldwork, and Susannah Buhrman-Deever for

232 providing data. This manuscript benefited from discussion with Dr. Alejandro Salinas-Melgoza,

233 Dr. Elizabeth Hobson, and Dominique Hellmich.

234

235 References:

236 1. Janik VM, Slater PJB. 2000 The different roles of social learning in vocal

237 communication. Anim. Behav. 60, 1-11. (doi:10.1006/anbe.2000.1410)

238 2. Nowicki S, Searcy W. 2014 The evolution of vocal learning. Curr. Opin. Neurobiol. 28,

239 48-53. (doi:10.1016/j.conb.2014.06.007)

240 3. Sewall KB, Young AM, Wright TF. 2016 Social calls provide novel insights into the

241 evolution of vocal learning. Anim. Behav. 120, 163-172.

242 (doi:10.1016/j.anbehav.2016.07.031)

243 4. Fripp D, Owen C, Quintana-Rizzo E, Shapiro A, Buckstaff K, Jankowski K, Wells R, Tyack P. 2005 Bottlenose dolphin (Tursiops truncatus) calves appear to model their signature whistles on the signature whistles of community members. Anim. Cogn. 8, 17-26. (doi:10.1007/s10071-004-0225-z) 
247 5. Berg KS, Delgado S, Cortopassi KA, Beissinger SR, Bradbury JW. 2012 Vertical transmission of learned signatures in a wild parrot. Proc. R. Soc. B Biol. Sci. 279, 585591. (doi:10.1098/rspb.2011.0932)

6. Berg KS, Delgado S, Okawa R, Beissinger SR, Bradbury JW. 2011 Contact calls are

7. Tibbetts EA, Dale J. 2007 Individual recognition: it is good to be different. Trends Ecol.

8. Janik VM, Sayigh LS, Wells RS. 2006 Signature whistle shape conveys identity used for individual mate recognition in free-ranging green-rumped parrotlets, Forpus passerinus. Anim. Behav. 81, 241-248. (doi:10.1016/j.anbehav.2010.10.012)

10. Lockwood JL, Cassey P, Blackburn T. 2005 The role of propagule pressure in explaining

11. Smith-Vidaurre G, Araya-Salas M, Wright TF. 2020 Individual signatures outweigh social information to bottlenose dolphins. Proc. Natl. Acad. Sci. U. S. A. 103, 8293-8297. (doi:10.1073/pnas.0509918103)

\section{1} group identity in contact calls of a communally nesting parrot. Behav. Ecol. 31, 448458. (doi:10.1093/beheco/arz202)

12. Russello MA, Avery ML, Wright TF. 2008 Genetic evidence links invasive monk parakeet populations in the United States to the international pet trade. BMC Evol. Biol. 8, 217. (doi:10.1186/1471-2148-8-217)

13. Buhrman-Deever SC, Rappaport AR, Bradbury JW. 2007 Geographic variation in contact calls of feral North American populations of the monk parakeet. Condor 109, 389-398. (doi:10.1525/boom.2013.3.4.67.B)

14. Bioacoustics Research Program. 2014 Raven Pro: Interactive Sound Analysis Software.

15. Araya-Salas M, Smith-Vidaurre G. 2017 warbleR: an R package to streamline analysis of animal acoustic signals. Methods Ecol. Evol. 8, 184-191. (doi:10.1111/2041210X.12624)

16. R Core Team. 2018 R: A Language and Environment for Statistical Computing.

17. Linhart P, Osiejuk TS, Budka M, Salek M, Spinka M, Policht R, Syrova M, Blumstein DT. 2019 Measuring individual identity information in animal signals: overview and performance of available identity metrics. Methods Ecol. Evol. 2019, 1558-1570. (doi:10.1111/2041-210X.13238) 
18. Parker KA, Anderson MJ, Jenkins PF, Brunton DH. 2012 The effects of translocationinduced isolation and fragmentation on the cultural evolution of bird song. Ecol. Lett. 15, 778-785. (doi:10.1111/j.1461-0248.2012.01797.x)

19. Lachlan RF, Verzijden MN, Bernard CS, Jonker PP, Koese B, Jaarsma S, Spoor W, Slater PJB, ten Cate C. 2013 The progressive loss of syntactical structure in bird song along an island colonization chain. Curr. Biol. 23, 1896-1901. (doi:10.1016/j.cub.2013.07.057)

20. Thielcke G. 1973 On the origin of divergence of learned signals (songs) in isolated populations. Ibis (Lond. 1859). 115, 511-516. (doi:10.1111/j.1474-919X.1973.tb01989.x)

21. Bock DG, Caseys C, Cousens RD, Hahn MA, Heredia SM, Hubner S, Turner KG, Whitney KD, Rieseberg LH. 2015 What we still don't know about invasion genetics. Mol. Ecol. 24, 2277-2297. (doi:10.1111/mec.13032)

22. Dlugosch KM, Parker IM. 2008 Founding events in species invasions: genetic variation, adaptive evolution, and the role of multiple introductions. Mol. Ecol. 17, 431-449. (doi:10.1111/j.1365-294X.2007.03538.x)

23. Edelaar P et al. 2015 Shared genetic diversity across the global invasive range of the monk parakeet suggests a common restricted geographic origin and the possibility of convergent selection. Mol. Ecol. 24, 2164-2176. (doi:10.1111/mec.13157)

24. Hobson EA, Avery ML, Wright TF. 2014 The socioecology of monk parakeets: insights into parrot social complexity. Auk 131, 756-775. (doi:10.1642/AUK-14-14.1)

25. Tibbetts EA, Liu M, Laub EC, Shen SF. 2020 Complex signals alter recognition accuracy and conspecific acceptance thresholds. Philos. Trans. R. Soc. B Biol. Sci. 375, 20190482. (doi:10.1098/rstb.2019.0482)

26. Bradbury JW, Balsby TJS. 2016 The functions of vocal learning in parrots. Behav. Ecol. Sociobiol. 70, 293-312. (doi:10.1007/s00265-016-2068-4)

27. Dooling RJ, Leek MR, Gleich O, Dent ML. 2002 Auditory temporal resolution in birds: discrimination of harmonic complexes. J. Acoust. Soc. Am. 112, 748-759.

(doi:10.1121/1.1494447) 